\title{
Cryogenic Performance of a Low-Noise JFET-CMOS Preamplifier for HPGe Detectors
}

\author{
Alberto Pullia, Francesca Zocca, Stefano Riboldi, Dusan Budjáš, Alessio D’Andragora, and Carla Cattadori
}

\begin{abstract}
Cryogenic low-noise charge sensitive preamplifiers have been developed and realized for the GERmanium Detector Array (GERDA). An integrated JFET-CMOS preamplifier, which is fully functional at cryogenic temperatures, has been tested in conjunction with an unsegmented p-type HPGe detector. Both the crystal and the preamplifier were operated inside a liquid nitrogen dewar at $77 \mathrm{~K}$. The detector capacitance was $\sim 60 \mathrm{pF}$. An optimum resolution of $1.6 \mathrm{keV}$ FWHM has been obtained for the pulser line at $6 \mu$ s shaping time. A resolution of $2.1 \mathrm{keV}$ FWHM has been achieved for the $1.332 \mathrm{MeV}$ line from a ${ }^{60} \mathrm{Co}$ source. A wide bandwidth (rise time of $\sim 16 \mathrm{~ns}$ ) permits use of pulse-shape analysis techniques to localize the position of the photon interactions inside the crystal. A low power consumption $(\sim 23 \mathrm{~mW})$ makes the preamplifier suitable for a multi-channel array of germanium detectors.
\end{abstract}

Index Terms-CMOS preamplifier, cryogenic low-noise preamplifier, gamma-ray spectroscopy, germanium detectors.

\section{INTRODUCTION}

$\mathbf{U}$ SE of High-Purity Germanium (HPGe) detectors is foreseen in the GERDA experiment (GERmanium Detector Array) [1], [2] in the search of neutrino-less double-beta decay of ${ }^{76} \mathrm{Ge}$. Bare germanium detectors, isotopically enriched in ${ }^{76} \mathrm{Ge}$, are operated in liquid argon. The cryogenic liquid is used both as a cooling medium and as a shield against external radiation. The charge preamplifiers must operate as completely immersed in the cryogenic liquid. Being an ultra-low background radiation detection system, the front-end electronics must also provide a high degree of radio-purity. Some different solutions have been studied and developed for the read-out of the GERDA crystals [3]-[5]. In particular a JFET-CMOS low-noise charge preamplifier has been developed, realized and tested. In this work the very good performances obtained are described. The circuit has been tested when coupled to an unsegmented coaxial HPGe crystal. Both the detector and the preamplifier were immersed in a liquid nitrogen dewar and operated at $77 \mathrm{~K}$. The

Manuscript received July 28, 2009; revised November 10, 2009. Current version published April 14, 2010.

A. Pullia, F. Zocca, and S. Riboldi are with the Department of Physics, University of Milano, 20133 Milano, Italy and also with INFN-Sezione di Milano, 20133 Milano, Italy (e-mail: alberto.pullia@mi.infn.it; francesca. zocca@mi.infn.it; stefano.riboldi@mi.infn.it).

D. Budjás is with the Max-Planck-Institut fuer Kernphysik, 69117 Heidelberg, Germany (e-mail: dusan.budjas@ mpi-hd.mpg.de).

A. D'Andragora is with the University of L'Aquila, 67010 L'Aquila, Italy and also with INFN-Laboratori Nazionali del Gran Sasso, 67010 L'Aquila, Italy (e-mail: alessio.dandragora@lngs.infn.it).

C. Cattadori is with the INFN-Sezione di Milano-Bicocca, 20133 Milano, Italy and also with INFN-Laboratori Nazionali del Gran Sasso, 67010 L'Aquila, Italy (e-mail: carla.cattadori@lngs.infn.it).

Digital Object Identifier 10.1109/TNS.2009.2038697 preamplifier performances meet the needed challenging requirements of functionality at cryogenic temperatures, low noise, low power consumption, rise time of $<20 \mathrm{~ns}$ and high closed-loop gain stability.

\section{Detector Setup AND Charge-PREAMPlifier Structure}

In Fig. 1 the cryogenic detector setup developed at Max-Planck-Institut fuer Kernphysik (MPIK) in Heidelberg [1] is shown. An unsegmented p-type coaxial closed-end HPGe crystal is encapsulated in a standard silicon holder and operated in a vacuum chamber (Fig. 1(a)). The crystal has a diameter of $5.2 \mathrm{~cm}$ and an height of $5.1 \mathrm{~cm}$. A plate for the mounting of the front-end electronics is placed below the vacuum chamber at a distance of $\sim 5 \mathrm{~cm}$ (Fig. 1(a)). Both the chamber and the plate are supported by a steel frame (Fig. 1(b)) so as to be directly immersed in a liquid nitrogen dewar (Fig. 1(c)). The germanium crystal is thermally connected to the cryostat (Fig. 1(a)) and therefore operated at $77 \mathrm{~K}$ as well as the front-end electronics. The detector outer contact is biased at the high voltage of 2.5 $\mathrm{kV}$. The inner contact is the read-out electrode and is DC-coupled to the preamplifier. As the read-out electrode receives holes, the signal polarity at the preamplifier output is negative.

In Figs. 2 and 3 the realized charge-sensitive preamplifier is shown. It is optimized for negative output signals and has the circuit structure shown in Fig. 2. It consists of an external low-noise silicon JFET (Junction Field Effect Transistor) manufactured by Philips, mod. BF862, an external feedback network $\left(C_{\mathrm{F}}=0.2 \mathrm{pF}, R_{\mathrm{F}}=1.2 \mathrm{G} \Omega\right.$ ), and an ASIC (Application Specific Integrated Circuit) used as low-noise operational amplifier along the negative-feedback loop. The ASIC has been realized in a $5 \mathrm{~V} 0.8 \mu \mathrm{m}$ silicon CMOS technology. The discrete input devices as well as the integrated circuit are mounted on a PCB of $0.8 \mathrm{~mm}$ PTFE (teflon) laminate, as shown in Fig. 3.

The design of an integrated preamplifier for high-resolution gamma-ray spectroscopy is particularly challenging. A large dynamic range of at least $70 \mathrm{~dB}$ is required, while in contrast the available voltage swing of scaled CMOS technologies is lower and lower. Moreover, a long-term gain stability better than $0.2 \%$ and a high integral linearity over the full range are required. The output stage is a very critical part as the signal is to be transmitted to a remote receiver through a long terminated coaxial cable. The output stage has to provide the peak power in the shortest possible time and it should be able to provide a rail-torail output voltage swing. A standard high output impedance solution is not particularly adequate for the present application as it makes the preamplifier loop gain highly dependent on the value of the output load resistor. A low output impedance 


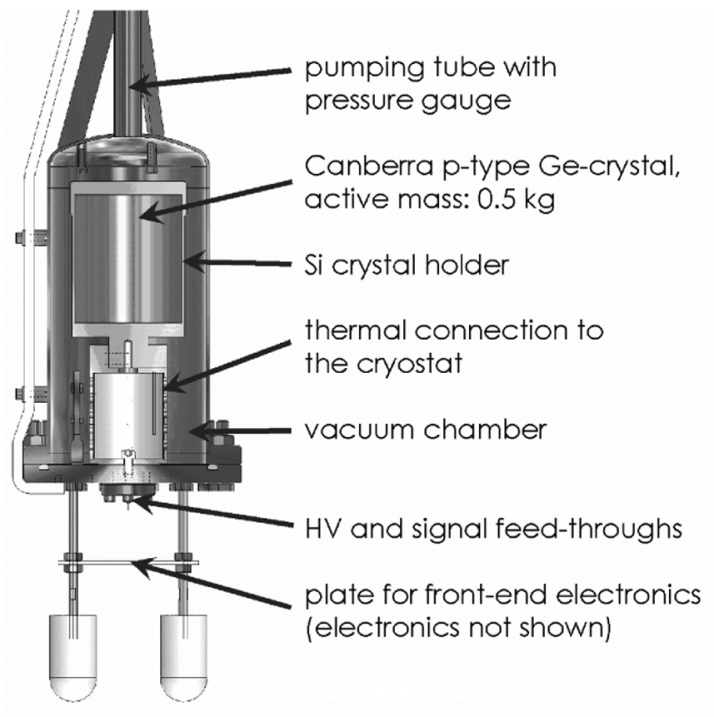

(a)

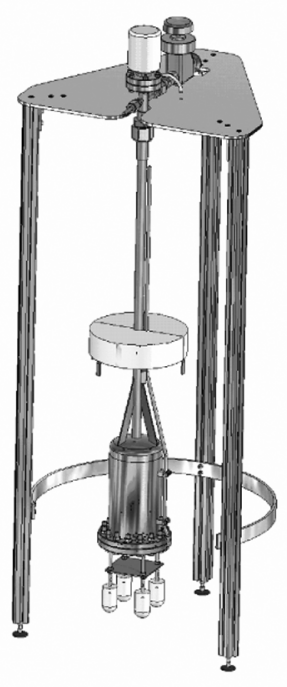

(b)

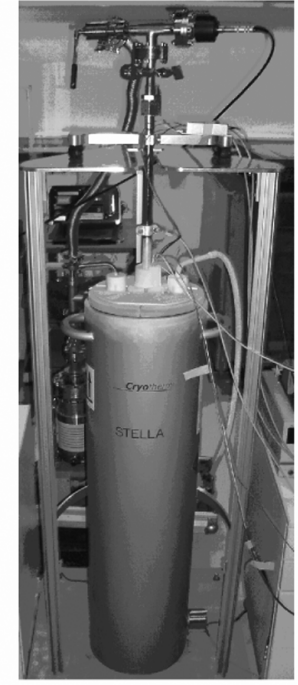

(c)

Fig. 1. Detector cryogenic set-up. (a) Vacuum chamber containing the encapsulated HPGe crystal. A plate for the mounting of the front-end electronics is placed below the chamber. (b) Steel frame supporting the vacuum chamber and the charge preamplifiers. (c) Liquid nitrogen dewar where the vacuum chamber and the charge preamplifiers are immersed and operated at $77 \mathrm{~K}$.

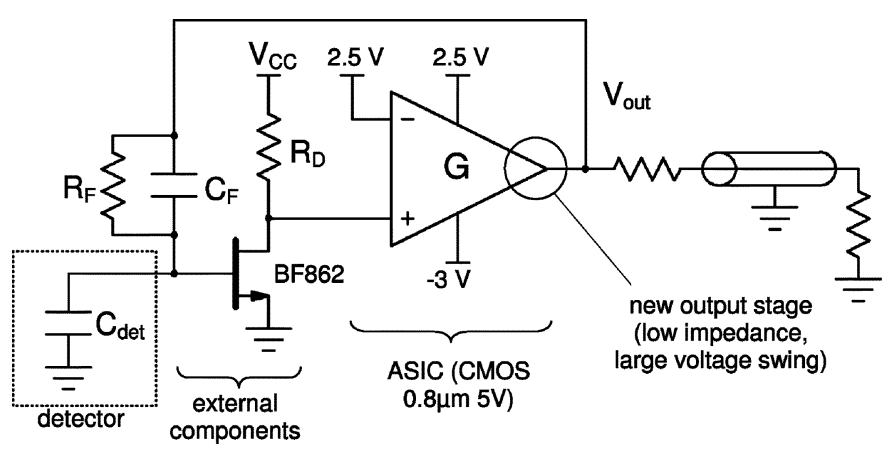

Fig. 2. Charge sensitive preamplifier structure, consisting of an external JFET, an external feedback network, and an ASIC used as low-noise operational amplifier along the negative-feedback loop.

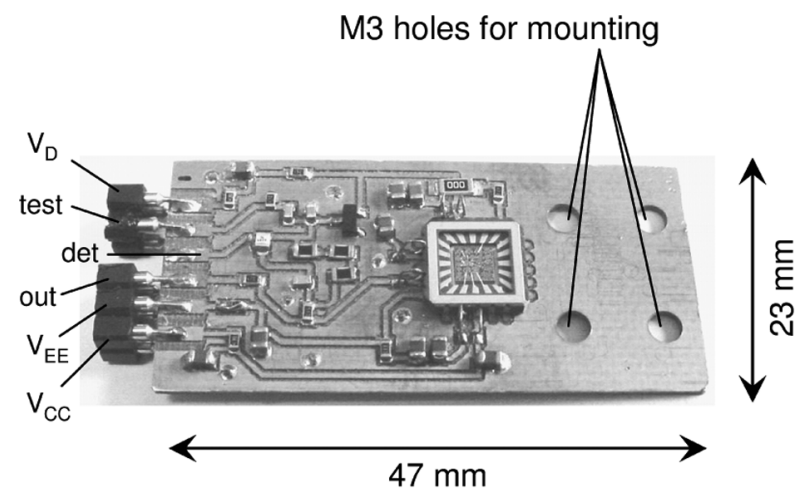

Fig. 3. Picture of the realized JFET-CMOS preamplifier as mounted on a PCB of $0.8 \mathrm{~mm}$ teflon laminate. The ASIC is realized in a $5 \mathrm{~V} 0.8 \mu \mathrm{m}$ silicon CMOS technology.

solution, optimized for negative signals (holes) has been realized, consisting of a self-adjusting constant current NMOS

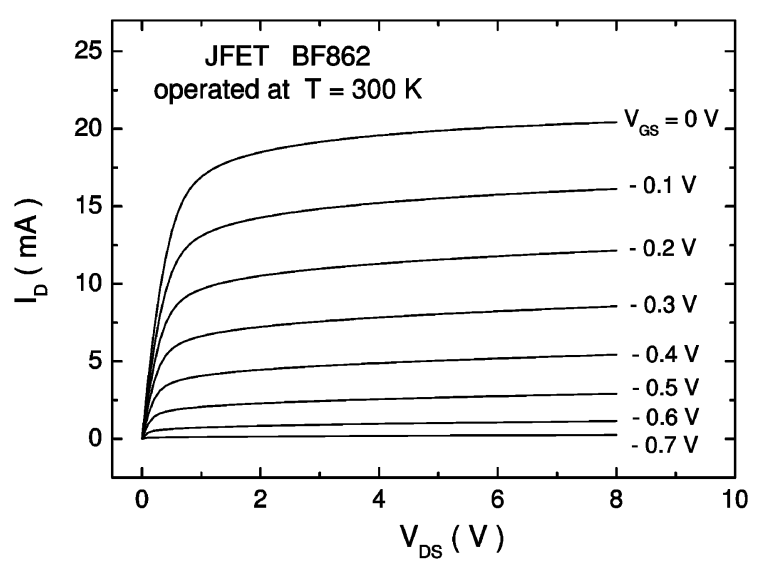

Fig. 4. Characteristic curves of the input Si-JFET BF862 as measured at room temperature.

source-follower. The current load is provided by a NMOS transistor acting as a driver. The detailed structure of the CMOS amplifier is described in [6], [7].

\section{Test-Bench Characterization of the PREAMPlifier AT $T=300 \mathrm{k}$ AND $T=77 \mathrm{k}$}

The preamplifier has been test-bench characterized, by simulating the detector with a capacitance and by injecting fast test pulses at the input node through a $1 \mathrm{pF}$ test capacitance. The characteristic curves of the input BF862 Si-JFET have been measured both at room temperature $(300 \mathrm{~K})$ and in liquid nitrogen $(77 \mathrm{~K})$. The results are shown in Figs. 4 and 5. The characteristic curves of the input JFET substantially change when the temperature is decreased from $300 \mathrm{~K}$ to $77 \mathrm{~K}$, as the freeze-out phenomena show up [8]. For given values of the gate-to-source and gate-to-drain voltages, the drain current decreases by a factor of $\sim 5$. The changed bias point at cryogenic temperature yields a remarkable loss of device performance, but 


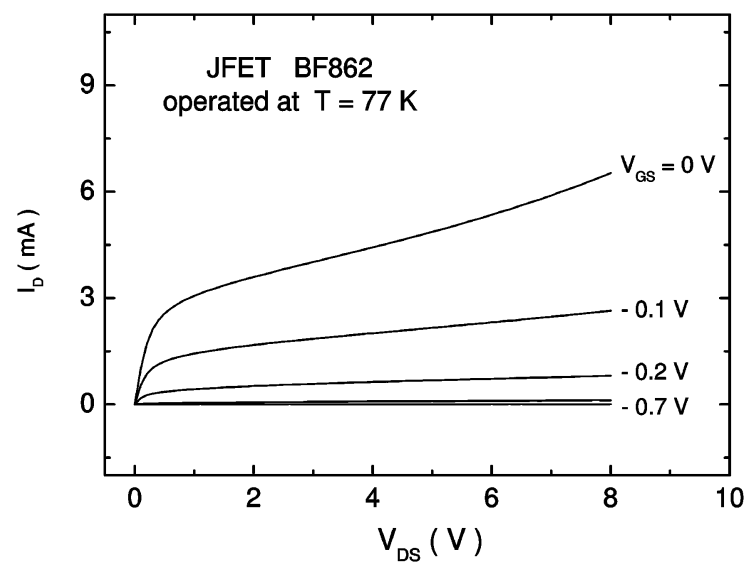

Fig. 5. Characteristic curves of the input JFET BF862 as measured at $77 \mathrm{~K}$, when the device is directly immersed in liquid nitrogen. The drain current decreases by a factor of $\sim 5$, yielding a substantial drop in the transconductance value.

the related drop of static power consumption is to be regarded as an advantage in this context. At room temperature the power supply voltage used for the external JFET is $12 \mathrm{~V}$ and the drain current is $14 \mathrm{~mA}$, so that the power consumption is $168 \mathrm{~mW}$. At $77 \mathrm{~K}$ the power supply voltage is $4 \mathrm{~V}$ and the drain current is $3 \mathrm{~mA}$, so that the power consumption is only $12 \mathrm{~mW}$. This reduces the liquid nitrogen micro-boiling phenomena as well as the related issues of microphonism; the cryogenic liquid consumption rate per unit preamplifier gets also substantially reduced. On the other hand, the changed JFET bias point determines a drop in the JFET transconductance, which can affect noise and bandwidth performances.

As far as noise is concerned, the Equivalent Noise Charge (ENC) of the circuit has been measured as a function of the shaping time, while simulating the detector with a capacitance of $15 \mathrm{pF}$, as shown in Fig. 6. The decreased temperature only partially compensates the effect of a decreased transconductance, so that the overall white noise is higher at $77 \mathrm{~K}$ than at room temperature. The optimum operating point for the input JFET would be at $120 \mathrm{~K}$, where the transconductance reaches the maximum value and the noise is at the minimum level [9], [10]. Unfortunately the front-end devices will not be operated at $120 \mathrm{~K}$ in the GERDA apparatus, which is the framework of this electronic development. Bare germanium detectors will be operated in a liquid argon cryostat having linear dimensions of several meters. The charge preamplifiers will be directly immersed in liquid argon at $86 \mathrm{~K}$. To let them operate at a higher temperature, a sealed vacuum box warming-up the front-end devices would be needed inside the cryostat, but the extremely low background radioactivity which is required in the proximity of the detectors does not allow for such a solution. Moving the front-end devices at a safer distance for radio-purity issues is not acceptable as well, because the long path from the detector electrodes to the input transistor would cause a substantial increase of the electronic noise and of pick-up disturbances and hence would completely spoil the spectroscopic performances. The choice of using all the same a Si-JFET as input transistor is dictated by the extremely low level of 1/f-like noise of such devices, which is particularly important for the present application. At a

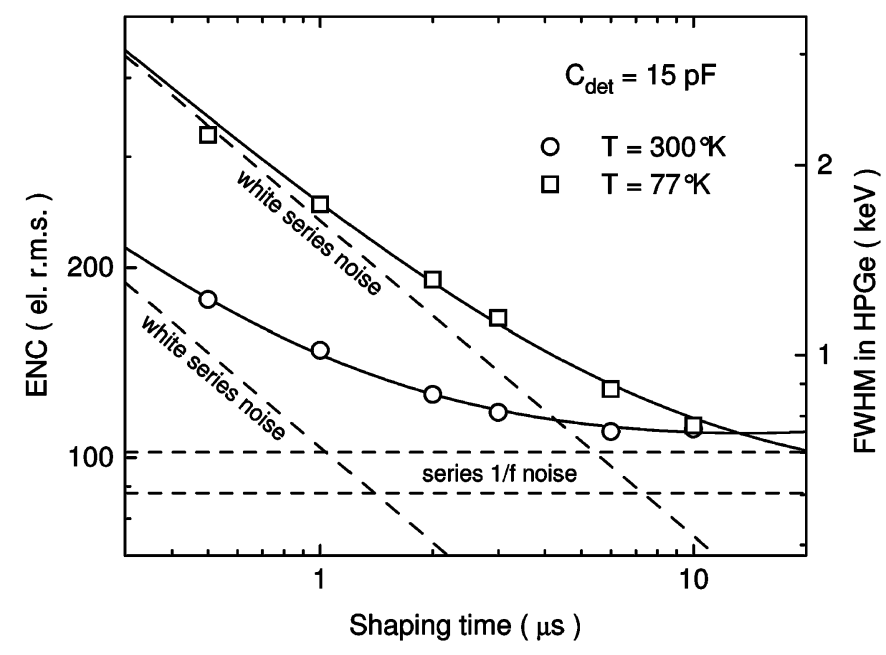

Fig. 6. Noise of the preamplifier as measured at the test bench, both at $300 \mathrm{~K}$ and $77 \mathrm{~K}$, as a function of the shaping time. The detector capacitance is $15 \mathrm{pF}$.

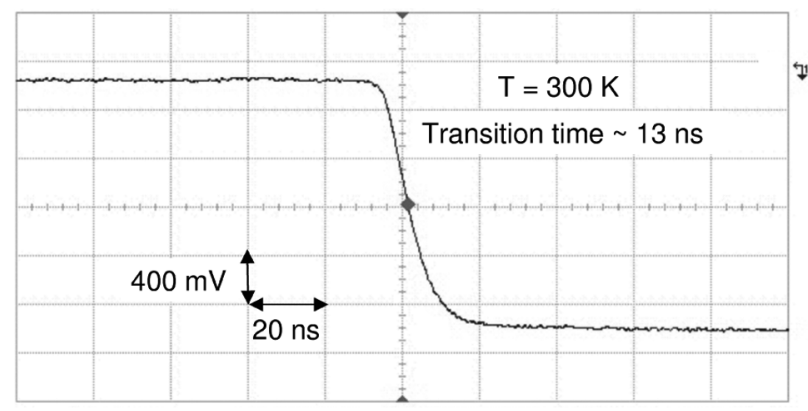

Fig. 7. Scope trace of the preamplifier response to a fast input pulse at $300 \mathrm{~K}$. The transition time is as fast as $13 \mathrm{~ns}$. The detector capacitance is $15 \mathrm{pF}$. The Miller compensation capacitance inside the ASIC is $0.6 \mathrm{pF}$.

shaping time of $10 \mu \mathrm{s}$, the ENC achieves the same value both at room and at cryogenic temperature, or $\sim 112$ electrons r.m.s. (corresponding to $0.77 \mathrm{keV}$ FWHM in HPGe). Hence, the noise performance remains fully compatible with gamma-ray spectroscopy requirements. The drop in the JFET transconductance at $77 \mathrm{~K}$ may be expected to determine a drop in the preamplifier bandwidth too. The opposite result is instead found: the preamplifier bandwidth increases when the circuit is operated at cryogenic temperature. The reason is easily found in the behavior of the transconductance of MOSFET transistors when operated at $77 \mathrm{~K}$. The MOSFET's transconductance definitely increases while temperature decreases. At low channel fields it is proportional to the low-field carrier mobility, which increases by a factor of 4 to 6 while decreasing the temperature from 300 to $77 \mathrm{~K}$, because of the reduced carrier scattering due to lattice vibration [11]. The higher gain of the ASIC amplifier at $77 \mathrm{~K}$ determines a higher loop gain of the overall preamplifier and a consequent wider bandwidth. While injecting a fast test pulse at the preamplifier input, a transition time of $13 \mathrm{~ns}$ is measured for the preamplifier response at room temperature, as shown in Fig. 7. When the circuit is operated in liquid nitrogen, the measured transition time is much faster but the output response shows some ringing. In order to get a fast but stable circuit response at cryogenic temperature, the preamplifier bandwidth has to be 
TABLE I

Transition Times Measured at the Test BeCh as Function of the DETECTOR CAPACITANCE (ASIC Miller CAPaCitANCE OF 1 PF)

\begin{tabular}{lcc}
\hline $\mathrm{C}_{\mathrm{DET}}$ & $\begin{array}{c}\text { Transition time @ } \\
\mathrm{T}=300 \mathrm{~K}\end{array}$ & $\begin{array}{c}\text { Transition time @ } \\
T=77 \mathrm{~K}\end{array}$ \\
\hline $0 \mathrm{pF}$ & $14 \mathrm{~ns}$ & $7 \mathrm{~ns}$ (ringing) \\
\hline $22 \mathrm{pF}$ & $21 \mathrm{~ns}$ & $12 \mathrm{~ns}$ (overshoot) \\
\hline $33 \mathrm{pF}$ & $26 \mathrm{~ns}$ & $13 \mathrm{~ns}$ \\
\hline $56 \mathrm{pF}$ & $34 \mathrm{~ns}$ & $18 \mathrm{~ns}$ \\
\hline $100 \mathrm{pF}$ & $50 \mathrm{~ns}$ & $28 \mathrm{~ns}$
\end{tabular}

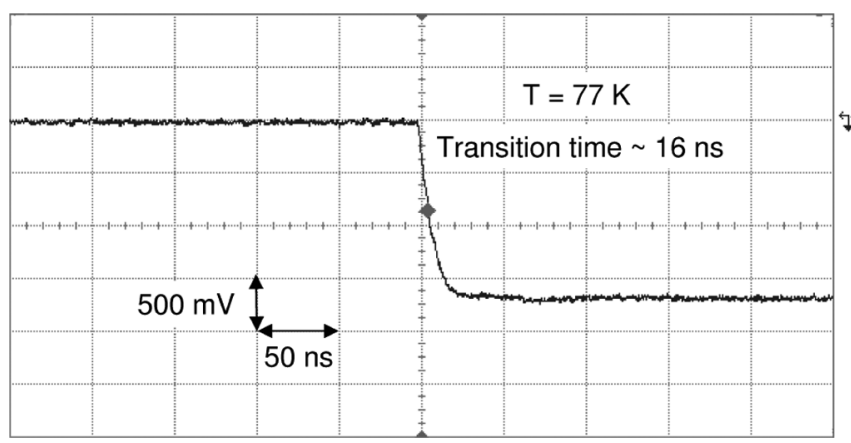

Fig. 8. Scope trace of the preamplifier output response to a fast input test pulse when the circuit is connected to the detector and operated at $77 \mathrm{~K}$. The transition time is as fast as $16 \mathrm{~ns}$.

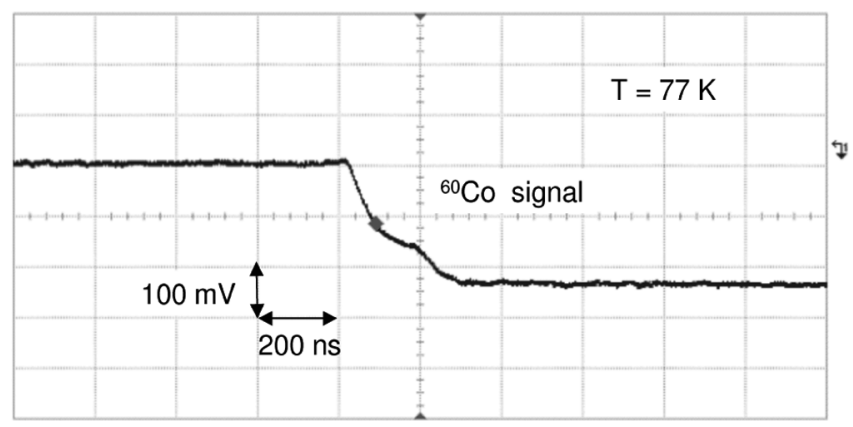

Fig. 9. Scope trace of a signal from a ${ }^{60} \mathrm{Co}$ radioactive source. The shape of the leading edge is well identified and permits application of pulse shape analysis algorithms.

reduced by increasing the value of the Miller compensation capacitance inside the ASIC amplifier, from a value of $0.6 \mathrm{pF}$ to 1 $\mathrm{pF}$. In Table I the measured transition time values are shown as obtained at $300 \mathrm{~K}$ and $77 \mathrm{~K}$ with a $1 \mathrm{pF}$ Miller capacitance. Note that the preamplifier bandwidth has been optimized in order to get a fast and stable response at $77 \mathrm{~K}$ with the highest expected values of the detector capacitance, at the expenses of a decreased bandwidth at room temperature.

\section{Experimental MeAsurements With THE Detector SETUP: PREAMPLIFIER PERFORMANCES AND ENERGY RESOLUTIONS}

The preamplifier has then been tested with the detector experimental setup described in Section II (see Fig. 1). In Figs. 8-12 some of the very good results obtained are shown.

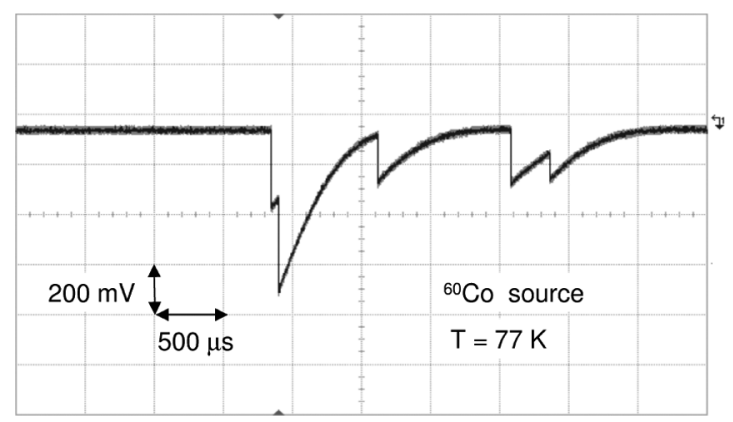

Fig. 10. Scope trace of a preamplifier output signal in the presence of a ${ }^{60} \mathrm{Co}$ source. The time constant of the baseline recovery is about $250 \mu \mathrm{s}$.

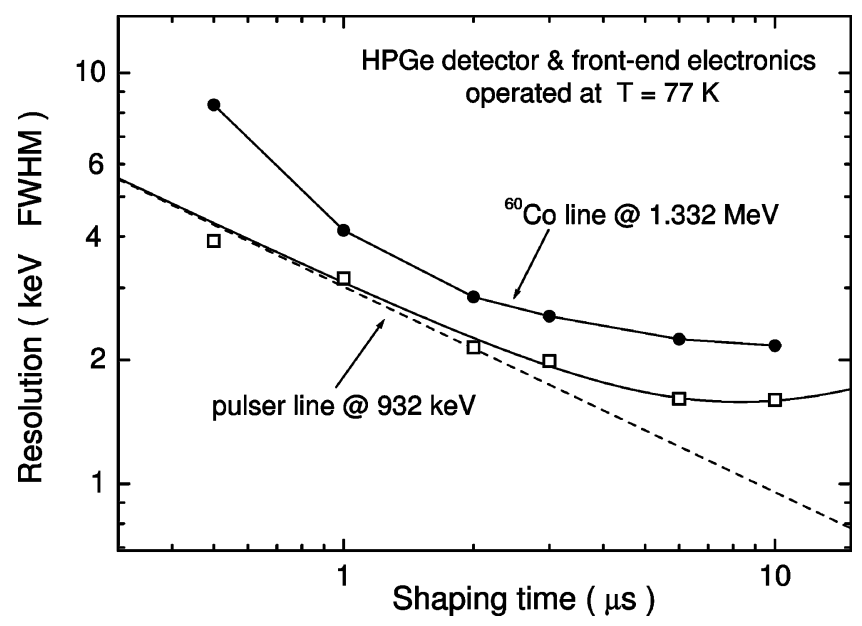

Fig. 11. Energy resolution as a function of the shaping time. The optimum resolution of $1.6 \mathrm{keV}$ FWHM is achieved for the pulser line at $6 \mu$ s shaping time. The detector capacitance is $\sim 60 \mathrm{pF}$.

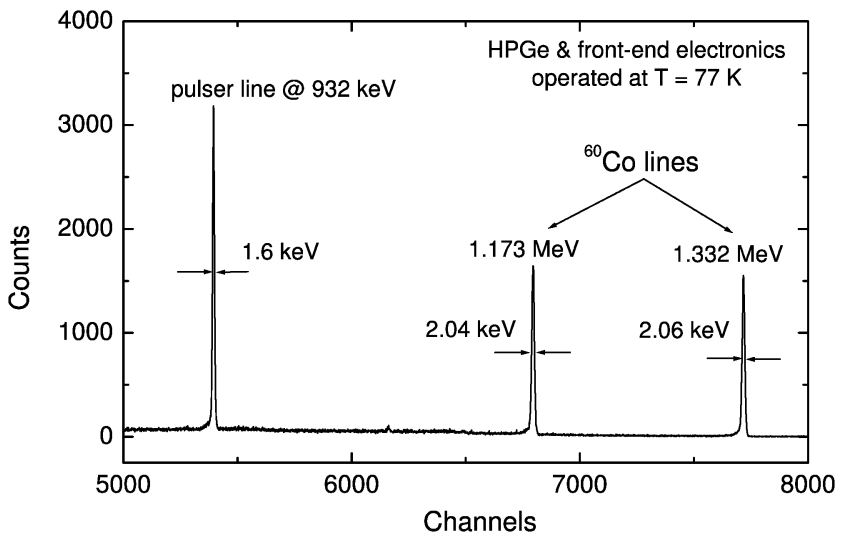

Fig. 12. Spectrum collected in the presence of a ${ }^{60} \mathrm{Co}$ source. A best resolution of 2.04 and $2.06 \mathrm{keV}$ FWHM has been obtained for the two ${ }^{60} \mathrm{Co}$ lines.

In Fig. 8 the preamplifier output response to a fast input test pulse is shown, when the circuit is connected to the detector and it is driving a $\sim 10 \mathrm{~m}$ coaxial output cable. A very clean leading edge is obtained with a transition time as fast as 16 ns. In Fig. 9 the leading edge of a signal from a ${ }^{60} \mathrm{Co}$ radioactive source is shown. Thanks to the wide preamplifier bandwidth, the shape of the leading edge is very well identified and permits application of pulse-shape analysis algorithms to localize the posi- 


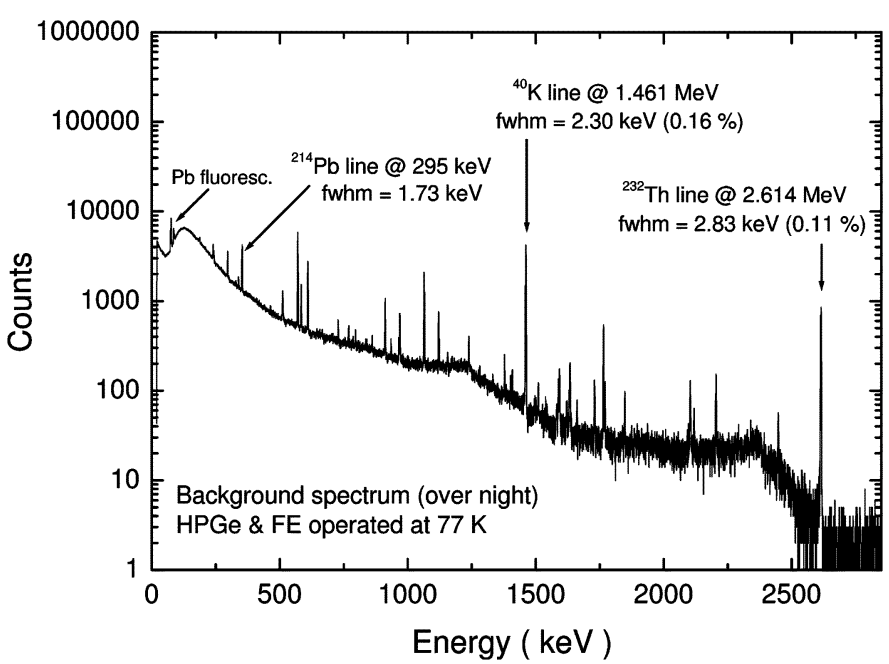

Fig. 13. Background spectrum collected over night. No peak shift or line broadening could be seen in long-term acquisitions.

tion of the photon interactions inside the crystal. In Fig. 10 the preamplifier output signal is shown in a longer time scale, when irradiating the detector with a ${ }^{60} \mathrm{Co}$ source. Note that the time constant of the baseline recovery is $\sim 250 \mu \mathrm{s}$. The energy resolution was then tested while connecting the preamplifier output to a quasi-Gaussian shaper amplifier and a pulse height analyzer. In Fig. 11 the obtained energy resolution is shown as a function of the shaping time for a pulser line and for the $1.332 \mathrm{MeV}^{60} \mathrm{Co}$ line. An optimum resolution of $1.6 \mathrm{keV} \mathrm{FWHM}$ is achieved for the pulser line at $6 \mu \mathrm{s}$ shaping time. The $1.332 \mathrm{MeV}{ }^{60} \mathrm{Co}$ line typically shows a resolution of $2.25 \mathrm{keV}$ FWHM at $6 \mu$ s and of $2.17 \mathrm{keV}$ FWHM at $10 \mu \mathrm{s}$. Note that the detector capacitance is $\sim 60 \mathrm{pF}$, while the input JFET capacitance is $\sim 10-15 \mathrm{pF}$. The choice of the BF862 model has been dictated by the need for an input device providing at the same time a very good noise performance and a very low power dissipation. Note also that the detector capacitance in the GERDA apparatus is estimated to be of $\sim 30 \mathrm{pF}$, so that a better capacitive matching will be finally achieved. The best collected ${ }^{60} \mathrm{Co}$ spectrum is shown in Fig. 12, where a very good resolution of 2.04 and $2.06 \mathrm{keV}$ FWHM has been achieved for the ${ }^{60} \mathrm{Co}$ lines. In Fig. 13 a background spectrum collected over night is shown. A resolution of $1.7 \mathrm{keV}$ FWHM or better is measured for the lines at the lowest energies. The limit in the achieved resolution corresponds to the electronic noise contribution. This is to be regarded as a good performance considering that the value for the detector capacitance is relatively high and that the naked preamplifier is working as directly immersed in liquid nitrogen, which is a very unusual operating condition. No peak shift or line broadening could be seen in this long-term acquisition, and very high resolutions, of the order of $0.1 \%$, could be achieved and maintained for the highest energy lines. A resolution of $2.30 \mathrm{keV}$ FWHM $(0.16 \%)$ has been measured for the background line of ${ }^{40} \mathrm{~K}$ at $1.461 \mathrm{MeV}$. A resolution of $2.83 \mathrm{keV}$ FWHM $(0.11 \%)$ has been achieved for the background line of ${ }^{232} \mathrm{Th}$ at 2.614 $\mathrm{MeV}$. The excellent stability of the line positions in long-term acquisitions confirms the very high loop gain of the preamplifier. A very good gain stability has also been observed when
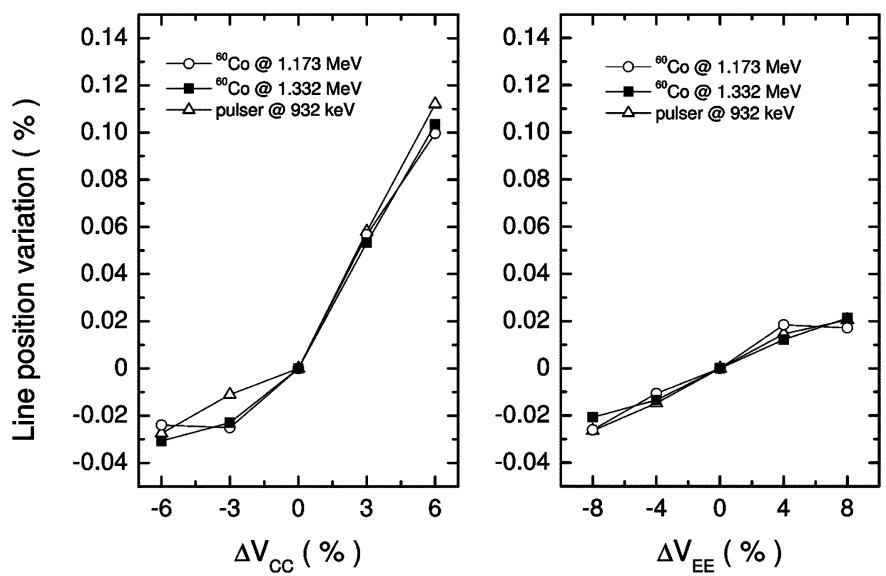

Fig. 14. Percent variation of the centroid positions of the spectrum lines as a function of the percent variation of the positive $\left(V_{\mathrm{CC}}\right)$ and negative $\left(V_{\mathrm{EE}}\right)$ power supply voltages of the ASIC.

TABLE II

PREAMPLIFIER SPECIFICATIONS

\begin{tabular}{|c|c|}
\hline Working temperature & $\begin{array}{l}\text { from }-196^{\circ} \mathrm{C} \text { to } 55^{\circ} \mathrm{C} \\
\text { (from } 77 \mathrm{~K} \text { to } 328 \mathrm{~K} \text { ) }\end{array}$ \\
\hline $\begin{array}{l}\text { Negative output voltage swing } \\
\text { on } 150 \Omega \text { impedance }\end{array}$ & $\begin{array}{l}\sim 2.5 \mathrm{~V} \text { (against a negative } \\
\text { power supply of }-2.7 \mathrm{~V} \text { ) }\end{array}$ \\
\hline $\begin{array}{l}\text { Energy sensitivity } \\
\qquad\left(C_{F}=0.2 p F\right)\end{array}$ & $\begin{array}{l}\sim 290 \mathrm{mV} / \mathrm{MeV} \text { at preamp } \\
\text { output } \\
\sim 217 \mathrm{mV} / \mathrm{MeV} \text { after } 150 \Omega \\
\quad \text { termination }\end{array}$ \\
\hline Input dynamic range & $\sim 8.6 \mathrm{MeV}$ \\
\hline Rise time at $\mathrm{T}=77 \mathrm{~K}$ & $\begin{array}{l}\sim 16 \text { ns with } \sim 10 \mathrm{~m} \text { terminated } \\
\text { coaxial cable }\end{array}$ \\
\hline Fall time at $\mathrm{T}=77 \mathrm{~K}$ & $\sim 250 \mu \mathrm{s}\left(\mathrm{R}_{\mathrm{F}}=1.2 \mathrm{G} \Omega\right)$ \\
\hline Open-loop gain at $\mathrm{T}=300 \mathrm{~K}$ & $3.5^{*} 10^{5}$ (Spice simulation) \\
\hline Loop gain at $T=300 \mathrm{~K}$ & 600 (Spice simulation) \\
\hline $\begin{array}{l}\text { Resolution at } \mathrm{T}=77 \mathrm{~K} \\
\qquad(\tau=6 \mu \mathrm{s})\end{array}$ & $\begin{array}{l}2.2 \mathrm{keV} @ 1.332 \mathrm{MeV}\left({ }^{60} \mathrm{Co}\right) \\
1.6 \mathrm{keV} @ 932 \mathrm{keV} \text { pulser line }\end{array}$ \\
\hline Power required at $\mathrm{T}=77 \mathrm{~K}$ & 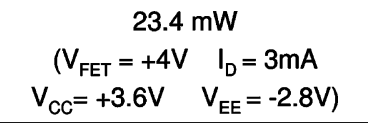 \\
\hline
\end{tabular}

measuring the line-centroid positions versus the value of the power supply voltages. In particular, as shown in Fig. 14, the rejection to changes in positive and negative power supply voltages of the ASIC circuit is shown. The position of the ${ }^{60} \mathrm{Co}$ lines, as well as that of the pulser line, show a typical stability of better than $0.1 \%$ against much larger percent variations, of up to $\pm 8 \%$, in the power supply voltages.

The preamplifier specifications are summarized in Table II. The negative output voltage swing is $\sim 2.5 \mathrm{~V}$, with a negative power supply of $-2.7 \mathrm{~V}$. As the preamplifier sensitivity is $\sim 290 \mathrm{mV} / \mathrm{MeV}$ (not terminated), the input dynamic energy range is $\sim 8.6 \mathrm{MeV}$. The quoted open-loop and loop gains have been obtained by a computer simulation at $300 \mathrm{~K}$. The available transistor Spice models have in fact a temperature range validity of $-40^{\circ} \mathrm{C}$ up to $125^{\circ} \mathrm{C}$, so that running a simulation at 
$-196^{\circ} \mathrm{C}(77 \mathrm{~K})$ is not allowed. The measured power consumption of the preamplifier is only $23.4 \mathrm{~mW}$. This is particularly important for future multi-channel arrays of germanium detectors, where a relatively high count of read-out channels inside the cryogenic liquid is foreseen.

\section{CONCLUSIONS}

A low-noise JFET-CMOS charge preamplifier for HPGe detectors has been realized and tested in the framework of the GERDA experiment. The circuit is functional both at room and cryogenic temperature and meets the requirements of high-resolution gamma-ray spectroscopy with germanium detectors. It has been tested in conjunction with an unsegmented p-type HPGe detector and operated inside a liquid nitrogen dewar at $77 \mathrm{~K}$. The detector capacitance is $\sim 60 \mathrm{pF}$. An optimum resolution of $1.6 \mathrm{keV}$ FWHM has been obtained on the pulser line at $6 \mu$ s shaping time. The $1.332 \mathrm{MeV}$ line from a ${ }^{60} \mathrm{Co}$ source shows a typical resolution of $2.2 \mathrm{keV}$ FWHM. A wide bandwidth (rise time of $\sim 16 \mathrm{~ns}$ ) and a low power consumption $(\sim 23 \mathrm{~mW})$ make the preamplifier suitable for multi-channel arrays of germanium detectors, where pulse-shape analysis techniques may be applied in order to localize the interaction positions of the gamma-photons inside the crystals.

\section{ACKNOWLEDGMENT}

The authors wish to thank R. Hofacker, S. Schönert, and H. Strecker for providing and setting up the SUB detector.

\section{REFERENCES}

[1] GERDA Home Page [Online]. Available: http://www.mpi-hd.mpg.de/ ge76

[2] S. Schonert et al., "GERDA: A new ${ }^{76}$ Ge double beta decay experiment at Gran Sasso," Nucl. Physics B (Suppl.), vol. 143, pp. 567-567, Jun. 2005.

[3] A. Pullia, F. Zocca, and C. Cattadori, "Low-noise amplification of $\gamma$-ray detector signals in hostile environments," IEEE Trans. Nucl. Sci., vol. 53, no. 3, pp. 1744-1748, Jun. 2006.

[4] C. Cattadori, O. Chkvorets, M. Junker, K. Kroeninger, L. Pandola, A. Pullia, V. Re, C. Tomei, C. Ur, and F. Zocca, "The GERmanium Detector Array read-out: Status and developments," Nucl. Instrum. Methods Phys. Res. A, vol. A572, pp. 479-480, Mar. 2007.

[5] S. Riboldi, A. Pullia, F. Zocca, D. Budjas, A. D' andragora, and C. Cattadori, "Test of a fully integrated CMOS preamplifier for HPGe detectors," in Proc. IEEE Nuclear Science Symp., Dresden, Germany, Oct. 19-25, 2008, pp. 2071-2073.

[6] A. Pullia, F. Zocca, and S. Riboldi, "A JFET-CMOS fast preamplifier for segmented germanium detectors," IEEE Trans. Nucl. Sci., vol. 55, no. 1, pp. 591-594, Feb. 2008.

[7] A. Pullia and F. Zocca, "A self-adjusting constant-current source follower for CMOS preamplifiers of semiconductor signals," in Proc. IEEE Nuclear Science Symp., San Diego, CA, Nov. 2006, vol. 1, pp. 354-356.

[8] R. T. Goldberg, M. D. Jhabvala, R. K. Kirschman, S. Wang, G.-H. Gwo, and J. A. Lipa, "Fabrication and characterization of low-noise cryogenic Si JFETs," in Proc. Symp. Low Temperature Electronics and High Temperature Superconductivity, Electrochemical Society, 1995, pp. 428-439.

[9] R. K. Kirschmann, "Low-temperature electronics," IEEE Circuits Devices Mag., vol. 6, pp. 12-24, 1990.

[10] N. C. Das, C. Monroy, and M. Jhabvala, "Germanium junction field effect transistor for cryogenic applications," Solid State Electron., vol. 44, pp. 937-940, 2000.

[11] W. F. Clark, B. El-Kareh, R. G. Pires, S. L. Titcomb, and R. L. Anderson, "Low temperature CMOS-A brief review," IEEE Trans. Compon., Hybrids, Manuf. Technol., vol. 15, pp. 397-404, 1992. 